\title{
The measurement of language lateralization with functional transcranial Doppler and functional MRI: a critical evaluation
}

\section{Metten Somers*, Sebastiaan F. Neggers, Kelly M. Diederen, Marco P. Boks, René S. Kahn and Iris E. Sommer}

Department of Psychiatry, Rudolf Magnus Institute of Neuroscience, University Medical Center Utrecht, Utrecht, Netherlands

\section{Edited by:}

Shuhei Yamaguchi, Shimane University, Japan

\section{Reviewed by:}

Hidenao Fukuyama, Kyoto University, Japan

Anke Bouma, University of Groningen, Netherlands

\section{${ }^{*}$ Correspondence:}

Metten Somers, Department of Psychiatry, University Medical Center Utrecht, B01.206, PO Box 85500, 3508 GA Utrecht, Netherlands. e-mail:m.somers@umcutrecht.n!
Cerebral language lateralization can be assessed in several ways. In healthy subjects, functional MRI (fMRI) during performance of a language task has evolved to be the most frequently applied method. Functional transcranial Doppler (fTCD) may provide a valid alternative, but has been used rarely. Both techniques have their own strengths and weaknesses and as a result may be applied in different fields of research. Until now, only one relatively small study $(n=13)$ investigated the correlation between lateralization indices (LIS) measured by fTCD and fMRI and showed a remarkably high correlation. To further evaluate the correlation between LIs measured with fTCD and fMRI, we compared LIs of 22 healthy subjects (12 left- and 10 righthanded) using the same word generation paradigm for the fTCD as for the fMRI experiment. LIs measured with fTCD were highly but imperfectly correlated with LIs measured with fMRI (Spearman's rho $=0.75, p<0.001$ ). The imperfectness of the correlation can partially be explained by methodological restrictions of $\mathrm{fMRI}$ as well as fTCD. Our results suggest that fTCD can be a valid alternative for $\mathrm{fMRI}$ to measure lateralization, particularly when costs or mobility are important factors in the study design.

Keywords: functional MRI, functional transcranial Doppler, cerebral blood flow measurement, language, cerebral lateralization, word generation task

\section{INTRODUCTION}

Language lateralization is the most thoroughly investigated aspect of functional cerebral lateralization (Sommer and Kahn, 2009). The direction of language lateralization, referred to as cerebral dominance, is related to hand-preference, as shown by studies applying the intra-carotid amobarbital procedure (IAP; Rasmussen and Milner, 1977; Isaacs et al., 2006), positron emission tomography (PET) studies (Tzourio et al., 1998), dichotic listening studies (Lewis et al., 1988), functional MRI (fMRI; Pujol et al., 1999; Springer et al., 1999; Szaflarski et al., 2002), and functional transcranial Doppler (fTCD) studies (Knecht et al., 2000b). The direction of language lateralization (cerebral dominance) is related to hand-preference. Approximately $95 \%$ of healthy right-handers have left-hemispheric dominance, whereas $70 \%$ of non-right-handers have left-hemispheric dominance, leaving $30 \%$ for right-hemispheric or bilateral dominance (Pujol et al., 1999; Szaflarski et al., 2002).

For the measurement of cerebral dominance, intra-operative brain mapping (Rutten et al., 2002a; Roux et al., 2003) and the IAP (Wellmer et al., 2008) remain the golden standards. However, due to their invasiveness these procedures are restricted to patients undergoing neurosurgery. The rapidly increasing body of literature on language lateralization that emerged over the last 15 years therefore largely stems from the application of non-invasive functional imaging techniques to measure language lateralization. A major part of these functional imaging studies has applied fMRI to assess lateralization. The fast rising popularity of $\mathrm{fMRI}$ is due to its high spatial resolution, the increasing availability of MRI scanners for research purposes, and its methodological validity for measuring real neuronal processing (Logothetis et al., 2001).
Lateralization indices (LIs) measured with fMRI have shown to be highly correlated to those measured with intra-operative brain mapping and the IAP (Binder et al., 1996; FitzGerald et al., 1997; Rutten et al., 2002a; Benke et al., 2006). In addition, fMRI provides information on localization of language activity within the hemispheres.

Functional MRI detects cerebral activity by measuring differences in the blood oxygenation level-dependent (BOLD) signal between different conditions (e.g., rest vs. performance of a language task). This signal change is measured in three-dimensional voxels that the brain can be partitioned into. The BOLD signal depends on three major contributions: blood flow, blood volume, and oxygen uptake (Ogawa et al., 1993; van Zijl et al., 1998). These factors all increase after neuronal activation due to the vasodilatation that results from neurovascular coupling (Buxton and Frank, 1997; Kim and Ugurbil, 1997; van Zijl et al., 1998). fMRI LIs (LI-fMRI) can be calculated by dividing the difference in the number of significantly activated voxels between hemispheres by the sum total of activated voxels. However, recent findings advocate calculating a LI based on differences in relative signal change (Jansen et al., 2006).

Functional MRI also has several disadvantages. Measurements are influenced by various non-neuronal variables, such as scanner drift and magnetic field in-homogeneities. Task related physiological factors such as movement, respiration, and heart rate changes further contribute to intra- as well as inter-subject variation (McGonigle et al., 2000; Zandbelt et al., 2008). Not surprisingly, test-retest studies for fMRI based language lateralization research indicated only moderate reproducibility (Rutten et al., 2002b; Jansen et al., 2006). 
Functional transcranial Doppler has been applied less frequently for the measurement of LIs, despite the high correlation with the golden standard intra-carotid amobarbital test (Knecht et al., 1998a; Rihs et al., 1999; Knake et al., 2003). FTCD allows for the determination of cerebral dominance by measuring changes in cerebral blood flow velocity (CBFV) in the right and left middle cerebral arteries (MCAs) during rest and during a word generation task (Deppe et al., 2004). The MCAs provide blood to a large region covering the lateral cortices of the brain, including the frontal, temporal, and parietal language areas (van der Zwan et al., 1993). During a language task, language areas of the dominant hemisphere will be more active than the contralateral areas, inducing an asymmetrical increase in CBFV in the MCAs. The difference in task related increase in CBFV between the left and right MCA can be used as a measure for cerebral dominance (Deppe et al., 2004). Degree and direction of cerebral dominance can be expressed by calculating a LI that describes the relative difference in increased cerebral blood flow between both hemispheres in subjects performing a language task. In addition to language lateralization research, fTCD has also been applied to investigate various cognitive measures ranging from general intelligence (Njemanze, 2005) to mathematical tests and face recognition (Droste et al., 1989) and the research of other lateralized functions such as attention (Floel et al., 2005) and spatial ability (Droste et al., 1989; Hartje et al., 1994; Vingerhoets and Stroobant, 1999).

Functional transcranial Doppler has some major advantages over scanner based neuroimaging techniques: the technique is much cheaper, more comfortable for the subject, easily applicable and its mobility allows measurements outside hospital or research institute settings for investigations of larger groups in the population.

The relative scarcity of studies applying fTCD to measure language lateralization may result from the lack of studies assessing reliability and validity of this technique. Thus far, only one study has shown LIs measured with fTCD and fMRI to be highly correlated $(r=0.95, p<0.0001$; Deppe et al., 2000). However, although this study had a solid design and was carried out accordingly, it was performed in a relatively small sample $(n=13)$ of strong left- and right-lateralized subjects. As such, the results might be an overestimation of the actual correlation between fTCD and fMRI. In addition, the word generation paradigms that were used in the fMRI and fTCD experiment used a different design. Furthermore, the fTCD and fMRI LIs that were compared in this study were based on different methods: a signal change based measure was used for fTCD LIs, while significant voxel count was used for the fMRI data.

To further investigate the correlation between LIs measured with fTCD and fMRI, we compared LIs of 22 healthy subjects (12 left- and 10 right-handed) using exactly similar word generation paradigms for the fTCD and the fMRI experiment and a signal change based LI for both fTCD and fMRI data.

\section{MATERIALS AND METHODS \\ SUBJECTS}

We included 22 healthy, native speakers from the UMC Utrecht environment ( 8 women and 14 men, mean age $=27.8, \mathrm{SD}=4.4$ ). Twelve were left-handed and 10 were right-handed according to a modified version of the Edinburgh Handedness Inventory (EHI-M; Oldfield, 1971; Isaacs et al., 2006). In the EHI-M participants indicate whether the listed activities are performed with the right-, left- or either hand, after which a LI can be calculated with the formula $(R-L) /(R+L) \times 100$. We aimed to include an equal number of right- and left-handers to increase the chance of finding a broad range of language LIs (Pujol et al., 1999; Szaflarski et al., 2002). Participants were between 18 and 50 years old and had no history of psychiatric or neurological disorder. Pregnant participants or those with metal objects in or around the body that could not be taken off were excluded. The study was approved by the Human Ethics Committee of the UMC Utrecht, Utrecht, The Netherlands. After a complete description of the study to the subjects, written informed consent was obtained according to the Declaration of Helsinki.

\section{FTCD EXPERIMENT: WORD GENERATION TASK}

For the fTCD experiment the procedures described by Deppe et al. (2004) were followed. After fixation of the headset and insonation of the MCAs, subjects were seated in front of a laptop computer. A word production paradigm used in several fTCD studies and validated by the IAP was displayed on a laptop computer screen (Knecht et al., 1996). Trials started with a 32.5-s baseline period during which the screen remained blank and subjects were instructed to think of non-verbal items (i.e., "a starry night"). At $t=32.5 \mathrm{~s}$, a cuing tone sounded, followed by a randomly picked letter at $t=37.5 \mathrm{~s}$ that was shown for $2.5 \mathrm{~s}$. Subjects were instructed to silently generate as many words as possible for $17.5 \mathrm{~s}$ starting with the displayed letter until the next cuing tone $(t=55 \mathrm{~s})$. Letters were shown only once. The letters Q, X, and Y were excluded, since these are infrequently used in Dutch language. Subjects were instructed to overtly repeat the last silently generated words after the second tone, to control performance. A third tone $(t=59 \mathrm{~s})$ indicated the end of a trial of which a total of 20 were performed.

\section{FTCD EXPERIMENT: DATA ACQUISITION}

A commercially available transcranial Doppler ultrasonography device (Multi-Dop T2, DWL, Sipplingen, Germany) was used for continuous measurements of changes in CBFV. MCAs were insonated at a depth of 40-55 mm with two 2-MHz transducer probes attached to a screw-top headset, after placing the probes bilaterally at the temporal skull windows (Deppe et al., 2004). FTCD spectral envelope curves were recorded and stored at a rate of 28 sample-points per second.

\section{FTCD EXPERIMENT: DATA ANALYSIS AND CALCULATION OF LATERALIZATION INDICES}

The AVERAGE software package was used for calculation of LI-fTCD by comparing changes in CBFV during covert word generation relative to resting state in both MCAs (Deppe et al., 1997). AVERAGE is designed to integrate fTCD data over the corresponding cardiac cycles. Data is consequently segmented into epochs time-locked to the cuing tone. These epochs can be averaged and analyzed to calculate LIs. A measure of the mean interhemispheric difference in $\mathrm{CBFV}$ is provided by subtracting averaged changes in CBFV during activity relative to rest $(\triangle \mathrm{CBFV})$ of the right and the left MCA. AVERAGE allows for calculation of the laterality index (LI-fTCD) by integrating the $\triangle \mathrm{CBFV}$ over a 2-s period of maximal difference between the left and right MCA during silent word generation. For an elaborate description of this methodology we 
refer to the paper by Deppe et al. (1997). A positive LI-fTCD reflects predominant left-hemispheric language processing. A negative sign reflects predominant right-hemispheric language processing. The magnitude of LI-fTCD reflects the degree of lateralization. In addition, AVERAGE calculates the accuracy of LI-fTCD, expressed by its confidence interval.

\section{FMRI EXPERIMENT: WORD GENERATION TASK}

For the fMRI experiment, the word generation task from the fTCD experiment was used. Subjects were additionally instructed to covertly repeat the last generated words after the second cuing tone to avoid movement artifacts. The task was precisely replicated in Presentation 11.3 (Neurobehavioural Systems, Albany, CA, USA), which was used to display the task in the scanner. Subjects wore headphones through which the cuing-tones could be heard. Although subjects could not be controlled for task performance, adequate cooperation was confirmed by their performance during the fTCD experiment.

\section{FMRI EXPERIMENT: DATA ACOUISITION}

Functional MRI data was obtained using a Philips Achieva 3 Tesla Clinical MRI scanner. The functional scan consisted of 1000 functional volumes and lasted $20.2 \mathrm{~min}$. Scans were acquired using Gradient-echo EPI with a $64 \times 64$ matrix and a voxel size of $4 \mathrm{~mm} \times 4 \mathrm{~mm} \times 4 \mathrm{~mm}$. The FOV was $256 \times 256 \times 80$, with a TE of $35 \mathrm{~ms}$ and a TR of $1212 \mathrm{~ms}$. Although this scan sequence does not cover the whole brain, it achieves coverage of large regions that are supplied by the MCAs and comprises all language areas. A T1 anatomical scan was performed for co-registration, consisting of 160 slices in a $256 \times 256$ matrix. There was no slice gap, slice thickness was $1 \mathrm{~mm}$. In-plane voxel size was $0.875 \mathrm{~mm} \times 0.875 \mathrm{~mm}$. The TE was $4.6 \mathrm{~ms}$, the TR $9.86 \mathrm{~ms}$, with a FOV of $224 \times 224 \times 160$.

\section{FMRI EXPERIMENT: PREPROCESSING AND DATA ANALYSIS}

Functional MRI data was analyzed using statistical parametric mapping (SPM5; Wellcome Department of Imaging Neuroscience, University College London, UK, http://www.fil.ion.ucl.ac.uk/ $\mathrm{spm} /$ ). By default, SPM convolves the neural input function (expected electrical neuronal activation) with a hemodynamic response function reflecting the delay in BOLD responses to brief electrical activation. In SPM5, the default choice for this convolution function is a superposition of 2 gamma functions (Henson and Friston, 2007), mimicking empirical observations of the neurovascular coupling (more options are available in SPM5). We used this default hemodynamic response model to convolve our on/off box-car function with. Preprocessing started with realignment to correct for effects of head motion. After co-registration, functional images were spatially normalized to a standard MNI template. Normalization parameters were determined on the T1 structural scan and subsequently applied to the functional images that were aligned with it in the previous steps. Images were smoothed using an 8-mm full-width-athalf-maximum (FWHM) Gaussian kernel. Although smoothing decreases spatial resolution, it increases the signal-to-noise ratio and is required for group analysis. Since we did not want the datasets to differ between the individual subject- and the group analysis, smoothed data was used for both.
Functional images were analyzed per participant on a voxel-byvoxel basis using multiple regression analysis (Worsley and Friston, 1995). A regression model was constructed with a one "box-car" factor coding blocks of covert word generation (on) and the rest periods in between (off). This model was fitted to the data per voxel, yielding regression coefficients, which were tested against 0 with a one sample $t$-test. This model tests whether activation is larger during task epochs as compared to the rest periods in between.

In addition, a second level random-effects analysis (one sample $t$-test) was conducted on the aforementioned regression coefficients ("beta-maps") to determine group-level activity of language areas (one sample $t$-tests, $p<0.05$ ). Activated language areas were identified using the same mask that was constructed for determination of LIs (see next paragraph). The threshold was set at 0.05 , with a voxel extent of 20 corrected for all voxels in the total brain by the false discovery rate (FDR; Benjamini and Hochberg, 1995). In the group analysis right- and left-handed subjects were pooled.

\section{FMRI EXPERIMENT: MAPPING OF LANGUAGE AREAS}

To calculate LIs from activations restricted to language areas, a mask comprising these areas was used. The mask was created using the automated anatomic labeling (AAL) atlas (Tzourio-Mazoyer et al., 2002) and included all cerebral areas that are thought to mediate language processing and their contralateral homologues (Sommer et al., 2008). The blood supply to these areas mainly relies on the MCAs (van der Zwan et al., 1993). As such, LI-fMRI based on activations restricted to language areas instead of the whole brain might more accurately correspond with LI-fTCD derived from CBVF changes in the MCA. The mask consisted of the triangular part of the inferior frontal gyrus, the insula, the middle temporal gyrus, the superior temporal gyrus, the supramarginal gyrus, and the angular gyrus. The mask was dilated by a factor 2 and co-registered to the normalized group analysis $t$-map. Due to the dilation, the mask comprised cortical areas outside the selected ones. Activated areas in the second level analysis were labeled using AAL (Tzourio-Mazoyer et al., 2002) running under SPM5.

\section{FMRI EXPERIMENT: CALCULATION OF LATERALIZATION INDICES}

Functional MRI lateralization indices (LI-fMRI) are traditionally calculated by comparing the number of significantly activated voxels in the left- and right-hemisphere and dividing this difference by the sum total of activated voxels (Binder et al., 1996). However, the use of signal intensity change has recently been shown a more robust and reproducible method than active voxel count methodology (Jansen et al., 2006). In addition, LI-fMRI based on signal change intensity might more accurately reflect cerebral blood flow changes than active voxel count methodology, hereby allowing a more accurate comparison with fTCD. As such, LIs were defined as the difference in signal intensity changes expressed by the sum total $\beta$-values in statistically suprathreshold voxels in the left- $\left(\beta_{\mathrm{LH}}\right)$ and the right-hemisphere $\left(\beta_{\mathrm{RH}}\right)$ within the selected language regions, divided by the sum total signal intensity changes in these voxels, expressed by the formula:

$$
\mathrm{LI}=\frac{\left[\sum\left(\beta_{\mathrm{LH}}\right)-\sum\left(\beta_{\mathrm{RH}}\right)\right]}{\left[\sum\left(\beta_{\mathrm{LH}}\right)+\sum\left(\beta_{\mathrm{RH}}\right)\right]}
$$


This formula yields LIs between 1, indicating strong left-hemisphere dominance and -1 , indicating strong right-hemisphere dominance. Thresholds were set at the individual $t$ values at $p=0.001$.

\section{ANALYSIS OF CORRELATION BETWEEN LI-fTCD AND LI-fMRI}

To calculate the correlation between LI-fTCD and LI-fMRI, Spearman's rho was calculated using SPSS 15.0, since LIs have been shown not to be distributed normally (e.g., Szaflarski et al., 2002). Correlations were calculated for the whole sample as well as for the subsamples of left- and right-handers. Using the Fisher $r$-to- $z$ transformation, we calculated the value of $\mathrm{z}$ to assess the two tailed significance of the difference between the two correlation coefficients.

\section{RESULTS}

\section{LATERALIZATION INDICES}

The overall correlation between LI-fTCD and LI-fMRI was significant, with Spearman's rho $=0.75, p<0.001$. See Figure 1 for a scatterplot of both measures over individuals. For an overview of fTCD, fMRI, and EHI-M LIs see Table 1. Separate analysis within the group of left-handed subjects also showed a high correlation between LI-fTCD and LI-fMRI, with Spearman's rho $=0.85, p=0.001$. Separate calculation of a correlation within right-handed subjects showed a non-significant Spearman's rho of $0.51, p=0.14$. There was no significant difference between the correlation coefficients of the subgroups of left- and right-handers $(Z=0.85, p=0.4)$.

\section{BRAIN ACTIVATIONS}

Group analysis of language activation showed activations of eight clusters, comprising several local maxima (see also Figure 2). In four out of eight clusters local maxima could be labeled using AAL

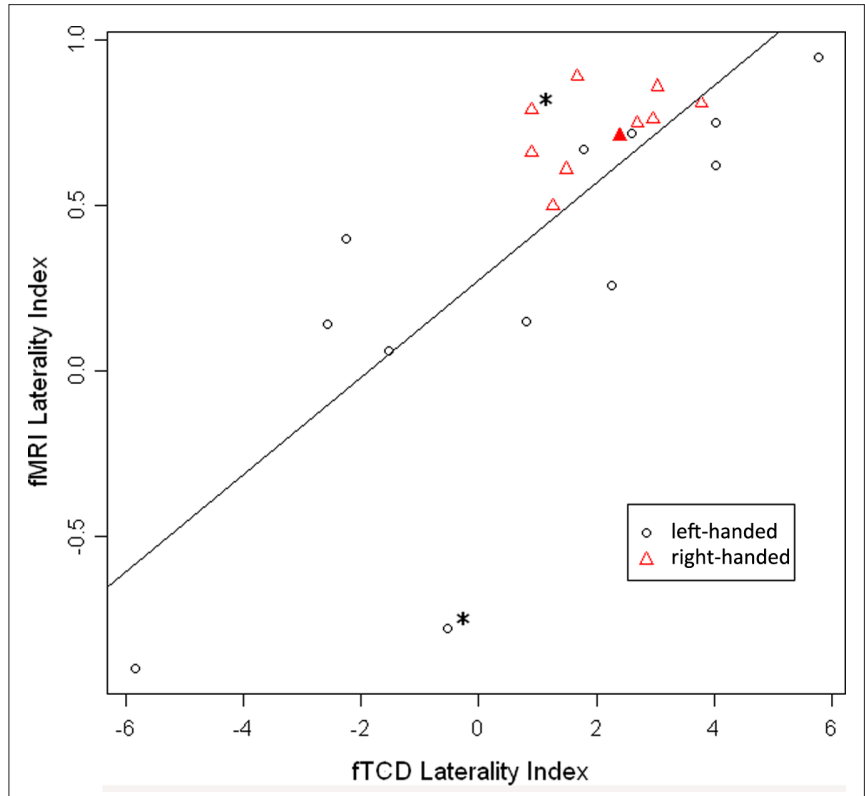

FIGURE 1 | Scatterplot of LI-fMRI $(-1<$ LI $<1)$ plotted against LI-fTCD $(-6<$ LI $<6)$. Red triangles: right-handed subjects. Black circles: left-handed subjects. Filled-out triangle: subject of which individual activation map is displayed (Figure 2B). "* " Indicates two subjects with an inaccurate fTCD measurement as shown by large confidence interval in Table 1 (subjects 5 and 17).
Table 1 | Lateralization indices of all subjects.

\begin{tabular}{|c|c|c|c|c|c|}
\hline Subject & Sex & LI-EHI-M & LI-fTCD & $\begin{array}{l}95 \% \text { Cl } \\
\text { LI-fTCD }\end{array}$ & LI-fMRI \\
\hline 1 & $\mathrm{~F}$ & 100 & 2.40 & 0.49 & 0.71 \\
\hline 2 & M & 100 & 0.89 & 0.44 & 0.66 \\
\hline 3 & F & 100 & 3.04 & 0.68 & 0.86 \\
\hline 4 & $M$ & 90 & 1.26 & 0.40 & 0.50 \\
\hline 5 & $\mathrm{~F}$ & 90 & 0.89 & 0.88 & 0.79 \\
\hline 6 & M & 90 & 3.79 & 0.81 & 0.81 \\
\hline 7 & $M$ & 80 & 2.96 & 0.53 & 0.76 \\
\hline 8 & M & 80 & 1.68 & 0.56 & 0.89 \\
\hline 9 & M & 70 & 2.70 & 0.82 & 0.75 \\
\hline 10 & F & 70 & 1.49 & 0.91 & 0.61 \\
\hline 11 & $\mathrm{~F}$ & -20 & 2.26 & 0.80 & 0.26 \\
\hline 12 & M & -40 & -2.25 & 0.62 & 0.40 \\
\hline 13 & M & -50 & 5.77 & 0.68 & 0.95 \\
\hline 14 & M & -60 & 1.78 & 0.63 & 0.67 \\
\hline 15 & $\mathrm{~F}$ & -80 & 4.02 & 0.46 & 0.62 \\
\hline 16 & F & -100 & 4.03 & 0.31 & 0.75 \\
\hline 17 & M & -100 & -0.53 & 1.44 & -0.78 \\
\hline 18 & M & -100 & -5.85 & 0.74 & -0.90 \\
\hline 19 & $\mathrm{~F}$ & -100 & 0.81 & 0.33 & 0.15 \\
\hline 20 & M & -100 & -2.58 & 0.58 & 0.14 \\
\hline 21 & M & -100 & -1.54 & 0.50 & 0.06 \\
\hline 22 & M & -100 & 2.61 & 0.38 & 0.72 \\
\hline
\end{tabular}

Individual subject data, showing sex, hand-preference lateralization indices (LI-EHI-M), fTCD lateralization indices (LI-fTCD), the 95\% confidence interval (95\% CI Ll-fTCD), and fMRI lateralization indices (LI-fMRI).

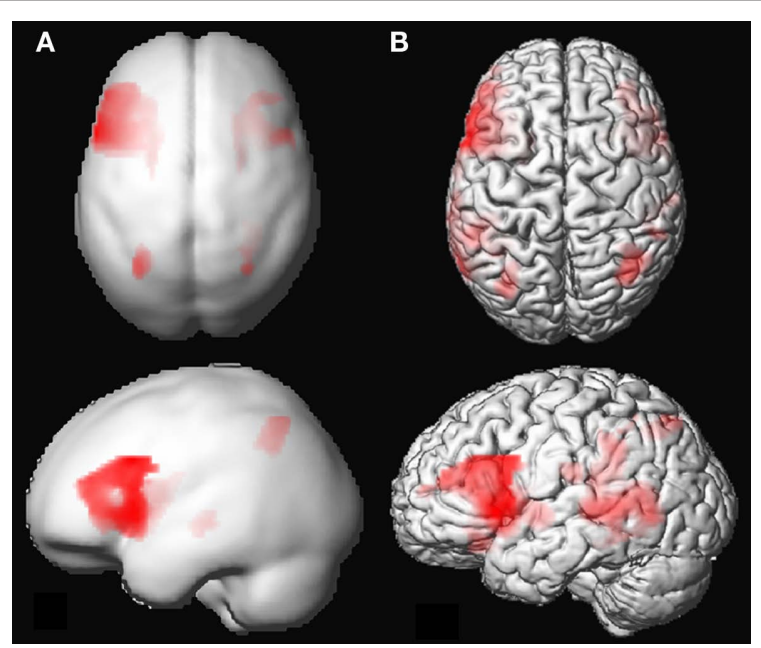

FIGURE 2 | (A) 3D rendering of language activation from the group analysis, intensity projected onto the gray-matter MNI space template from SPM5. FDR corrected (threshold 0.05) for the whole brain, voxel extent $=20$. For labeling of activated areas, see Table 2. (B) Activation map of 1 representative individual subject, intensity projected on normalized gray-matter rendering of the subject's T1 scan. LI-fMRI $=0.75$, LI-fTCD $=2.70$.

(Table 2). The first cluster showed activity in the left pre-central gyrus, the left putamen, and the triangular part of the left inferior frontal gyrus. The second cluster showed activity in the right putamen. The third cluster showed activity in the left inferior parietal 
Table 2 | Functional MRI activation coordinates in MNI space and AAL label.

\begin{tabular}{|c|c|c|c|c|c|c|}
\hline Cluster \# & $x$ & $y$ & $z$ & \# Voxels & z-score & Structure \\
\hline 1 & -46 & 12 & 30 & 3889 & 5.67 & $\begin{array}{l}\text { Left pre-central } \\
\text { gyrus }^{1}\end{array}$ \\
\hline 1 & -22 & 4 & 8 & & 5.31 & Left putamen ${ }^{1}$ \\
\hline 1 & -44 & 20 & 24 & & 5.25 & $\begin{array}{l}\text { Triangular part } \\
\text { of left inferior } \\
\text { frontal gyrus }{ }^{1}\end{array}$ \\
\hline 2 & 34 & 20 & 0 & 1248 & 5.31 & $\begin{array}{l}\text { Right inferior } \\
\text { frontal gyrus/ } \\
\text { insula } 2^{2}\end{array}$ \\
\hline 2 & 26 & 2 & 8 & & 4.85 & Right putamen' \\
\hline 2 & 48 & 12 & -4 & & 4.81 & Right insula ${ }^{1}$ \\
\hline 3 & 38 & -40 & 4 & 230 & 4.80 & White matter ${ }^{2}$ \\
\hline 4 & -30 & -26 & -4 & 64 & 4.55 & White matter ${ }^{2}$ \\
\hline 5 & -30 & -60 & 48 & 221 & 3.87 & $\begin{array}{l}\text { Left inferior } \\
\text { parietal lobule }\end{array}$ \\
\hline 5 & -30 & -60 & 38 & & 3.69 & $\begin{array}{l}\text { Left inferior } \\
\text { parietal lobule }{ }^{1}\end{array}$ \\
\hline 6 & 36 & 34 & 16 & 78 & 3.77 & White matter ${ }^{2}$ \\
\hline 7 & 28 & -52 & 36 & 26 & 3.35 & White matter ${ }^{2}$ \\
\hline 8 & 30 & -64 & 50 & 27 & 3.19 & $\begin{array}{l}\text { Right superior } \\
\text { parietal lobule }\end{array}$ \\
\hline
\end{tabular}

'Overview of all large clusters of activity, showing the total number of activated voxels and coordinates of local maxima at a threshold of 0.05 and voxel extent of 20, whole-brain FDR corrected. Of those clusters that could be labeled with the automated anatomical atlas (AAL) structure names are shown as identified by the AAL.

${ }^{2}$ Structures in clusters that could not be labeled with the AAL were identified on visual inspection.

lobule, the fourth cluster showed activity in the right parietal lobule. The remaining clusters could not be labeled with AAL. On visual inspection, one of these clusters was located in the right inferior frontal gyrus and insula. The four remaining clusters were located in white matter. For an overview, see Table 2.

\section{DISCUSSION}

This study investigated the correlation between LIs measured by fMRI and fTCD using a similar task paradigm in 22 subjects. We found a high overall correlation between fTCD lateralization indices (LI-fTCD) and fMRI lateralization indices (LI-fMRI; rho $=0.75, p<0.001)$. In left-handers, the correlation index was slightly higher ( $\mathrm{rho}=0.85, p=0.001$ ) despite the stronger inconsistency in degree of language lateralization (see subjects $11,12,17$, 20, and 21, Table 1) in comparison to right-handers. Our results show that LIs measured by fTCD and fMRI were highly correlated. These findings are in line with the previous findings of Deppe et al. (2000). Earlier studies that compared LIs measured with fTCD and the intra-carotid amobarbital test (IAP; Knecht et al., 1998a; Rihs et al., 1999; Knake et al., 2003) and LIs measured with fMRI and the IAP (e.g., Binder et al., 1996) found correlations of comparable strength.

Although our findings show a high overall correlate between LI-fTCD and LI-fMRI, this is considerably lower than the 0.95 correlation reported by Deppe et al. (2000). There are several potential explanations for this discrepancy.
The sample in the paper of Deppe et al. (2000) consisted of 13 subjects that were selected from a sample of 188 subjects with known LI-fTCD. These subjects all displayed strong right- or lefthemispheric lateralization. As a result, LIs were evenly distributed over both hemispheres and the two methods were found to be highly correlated. However, when measuring strong lateralized subjects only, the strength of the resulting correlation might overestimate the actual relation between the two methods. In our study subjects were selected for hand-preference without a priori knowledge of cerebral dominance. This resulted in a right shifted distribution of LIs, with a restricted range of indices in right-handers and several, mainly left-handed subjects with a LI around zero. This higher number of low lateralized subjects might partially account for the imperfect correlation in our study, since low lateralization is more difficult to measure reliably than strong lateralization (Jansen et al., 2006). Such difficulties with measuring low lateralization might be present in fTCD as well as fMRI. However, the small number of bilateral subjects in our sample precludes firm conclusions about this issue.

Difficulties in measuring low lateralization with fTCD have not been extensively investigated. However, for two subjects displaying a low LI-fTCD (subjects 17 and 5, Table 1 and Figure 1), the fTCD measurement might have been inaccurate as indicated by a large confidence interval relative to the LI-fTCD.

With fMRI, difficulties with the measurement, interpretation, and reproducibility of low lateralization have been clearly demonstrated. In a previous fMRI study, several subjects displayed bilateral activity even when the IAP indicated strong cerebral dominance (Woermann et al., 2003). This might result from activations in cortical areas that are engaged in language, but do not have a critical function (Desmond and Chen, 2002). Bilaterality on fMRI can also result from varying statistical thresholds (Rutten et al., 2002b). While increasing thresholds generally leads to higher LIs, lowering thresholds can increase bilaterality by raising the number of suprathreshold voxels in both hemispheres. It has also been shown that an increase of bilaterality might stem from task repetition induced changes in autonomic drive, leading to an increase of bihemispheric blood flow (Lohmann et al., 2004). An additional problem is that the reproducibility of fMRI LIs has been shown to be the lowest in subjects with bilateral language activity (Adcock et al., 2003; Jansen et al., 2006).

Low reproducibility is, however, not restricted to bilateral subjects, as overall fMRI reproducibility of LIs has almost invariably been shown to be low. For example, Fernandez et al. (2003) found that maximal $48.9 \%$ of activated voxels overlapped between two measurements. In their study investigating the reproducibility of LIs, Jansen et al. (2006) found that the highest Pearson correlation coefficient between two measurements of subjects performing a word generation task ranged from 0.61 to 0.66 when LIs were calculated by signal intensity changes. In contrast, fTCD has shown a high test-retest reliability, with a Pearson product moment correlation coefficient of $r=0.95$ obtained in a sample of 10 subjects (Knecht et al., 1998b). These differences in reproducibility might reflect technical differences between fTCD and fMRI that make the a priori chance of finding a near-perfect correlation between the two techniques unlikely. For example, the fTCD signal only depends on neurovascular-coupled CBFV changes, since the main 
confounding variable, heart-frequency variation, is corrected for in the AVERAGE software package (Deppe et al., 1997). In contrast, fMRI measures the vascular (BOLD) response to neuronal activation, mainly a rapid vasodilatation, through the neurovascular coupling. The increase in BOLD signal after neuronal activation results from a combination of changes in CBFV, cerebral blood volume, and cerebral metabolic rate of oxygen consumption $\left(\mathrm{CMRO}_{2}\right.$; see Kwong et al., 1992 for an overview). Since there is consensus that changes in CBFV constitute a major part of the BOLD response (Fox and Raichle, 1986), it is reasonable to assume that fTCD and fMRI BOLD correlate significantly. However, due to the aforementioned contributions to the BOLD signal other than CBFV a near-perfect correlation with fTCD is unlikely, which corresponds with our present findings.

In addition to this, the BOLD fMRI signal is influenced by various non-neuronal variables such as scanner drift, magnetic field inhomogeneities, and task related physiological factors (McGonigle et al., 2000; Zandbelt et al., 2008). Finally, fMRI data quality is influenced by various data acquisition parameters. For example, fMRI reproducibility has been shown to increase as a function of the number of volumes collected (Genovese et al., 1997).

Another possible confounder might be the different context in which the task is carried out in the fTCD and the fMRI experiment. While the fTCD experiment is performed in a quite natural environment (a silent room) this is not the case for fMRI, where the subject is lying in the scanner tunnel. In addition, fMRI acoustic noise has been shown to affect brain activation patterns (Tomasi et al., 2005).

Further, the task that was applied in this experiment engages a number of non-language related processes, such as spatial attention, working memory, and arousal, that might engage right-hemispheric activity (Knecht et al., 1996). As such, our LIs might be blurred by non-language related right-hemispheric activations, which can contribute to the imperfectness of the correlation. A further contribution to this imperfectness could result from possible taskrepetition effects and between-subject differences with regards to recruitment of neural resources and task-performance strategies.

In fTCD, LIs are calculated from relative changes in CBFV, while the majority of fMRI studies, including the earlier fMRI-fTCD comparison study of Deppe et al. (2000), use active voxel count methodology. In contrast, the present study adopted a relative signal intensity change based method for calculation of LI-fMRI that might more accurately reflect changes in cerebral perfusion and allows a more direct comparison with LI-fTCD. As such, the correlation we observed in this study is likely to be a realistic reflection of blood flow changes in both techniques.

Although both fMRI and fTCD allow for the calculation of LIs and have a comparable excellent temporal resolution, either technique has its own specific applicability and restrictions. One of the

\section{REFERENCES}

Adcock, J. E., Wise, R. G., Oxbury, J. M., Oxbury, S. M., and Matthews, P. M. (2003). Quantitative fMRI assessment of the differences in lateralization of language-related brain activation in patients with temporal lobe epilepsy. Neuroimage 18, 423-438.
Benjamini, Y., and Hochberg, Y. (1995). Controlling the false discovery rate. A practical and powerful approach to multiple testing. J. R. Stat. Soc., Ser. B Stat. Methodol. 57, 289-300.

Benke, T., Koylu, B., Visani, P., Karner, E., Brenneis, C., Bartha, L., Trinka, E., Trieb, T., Felber, S., Bauer, G.,

major benefits of fMRI is the high spatial resolution, that allows for the investigation of cortical activity on a neuroanatomical level (Logothetis et al., 2001). As such, fMRI might be the technique of choice when investigating the neural correlates of language function. In contrast, fTCD measurements are restricted to CBFV changes in the MCAs (Deppe et al., 2004). As a result, findings can only be interpreted at the level of hemispheric processing and not at a neuroanatomical level. This might give the impression that fTCD applicability seems constrained in comparison to fMRI. However, there are several benefits to fTCD in comparison to fMRI. First, the accuracy of fTCD measurements can be easily determined by the confidence interval of a measurement (Knecht et al., 1998b). The low costs, mobility, and easy and fast applicability with regards to data collection as well as analysis make fTCD an ideal technique for large scale studies, that, for example, allows for the collection of language lateralization data from population samples (e.g., Knecht et al., 2000a). Apart from language lateralization research, fTCD can also be applied for the large scale investigation of non-language functions, such as spatial attention (Floel et al., 2005) or spatial ability (Vingerhoets and Stroobant, 1999). Finally, besides this applicability in fundamental research questions, the characteristics of fTCD also make it an excellent tool to investigate patients in a clinical setting. This might even be enhanced by the previously mentioned fact that fTCD measurements can take place in a more natural environment than fMRI. One disadvantage of fTCD is that measurements are not possible in approximately $5 \%$ of subjects due to temporal bone window thickness (Knecht et al., 2000b; Deppe et al., 2004). The latter has been shown to differ between races and gender, with white males showing the least and black females showing the greatest bone thickness (Halsey, 1990).

A possible limitation of our study is that the two different methods of measuring language lateralization were not applied in a fully counterbalanced fashion. Further, performance during the fMRI scan could not be measured, since subjects were not allowed to speak in the scanner in order to reduce head motion. However, as mentioned earlier, subjects performed the fTCD task under supervision and all showed good cooperation. Still, in future fMRI studies the application of an adequate performance measure should be an important point of attention.

In conclusion, LIs measured by fTCD and fMRI show a robust yet imperfect correlation. The imperfectness of this correlation can be partially explained by methodological inconsistencies, especially with regards to fMRI, and, notably, by the profound differences in the neurophysiological signals underlying both measures (see Discussion above). Although both techniques have their own specific applicability, fTCD can be an excellent tool for large scale investigations of language lateralization and other cognitive functions, in healthy subjects as well as patients.

Chemelli, A., and Willmes, K. (2006) Language lateralization in temporal lobe epilepsy: a comparison between fMRI and the Wada Test. Epilepsia 47, 1308-1319.

Binder, J. R., Swanson, S. J., Hammeke, T. A., Morris, G. L., Mueller, W. M., Fischer, M., Benbadis, S., Frost, J.
A., Rao, S. M., and Haughton, V. M. (1996). Determination of language dominance using functional MRI: a comparison with the Wada test. Neurology 46, 978-984.

Buxton, R. B., and Frank, L. R. (1997). A model for the coupling between cerebral blood flow and oxygen 
metabolism during neural stimulation. J. Cereb. Blood Flow Metab. 17, 64-72.

Deppe, M., Knecht, S., Henningsen, H., and Ringelstein, E. B. (1997). AVERAGE: a Windows program for automated analysis of event related cerebral blood flow. J. Neurosci. Methods 75, 147-154.

Deppe, M., Knecht, S., Papke, K., Lohmann, H., Fleischer, H., Heindel, W., Ringelstein, E. B., and Henningsen, H. (2000). Assessment of hemispheric language lateralization: a comparison between fMRI and fTCD. J. Cereb. Blood Flow Metab. 20, 263-268.

Deppe, M., Ringelstein, E. B., and Knecht, S. (2004). The investigation of functional brain lateralization by transcranial Doppler sonography. Neuroimage 21, 1124-1146.

Desmond, J. E., and Chen, S. H. (2002). Ethical issues in the clinical application of fMRI: factors affecting the validity and interpretation of activations. Brain Cogn. 50, 482-497.

Droste, D. W., Harders, A. G., and Rastogi, E. (1989). A transcranial Doppler study of blood flow velocity in the middle cerebral arteries performed at rest and during mental activities. Stroke 20, 1005-1011.

Fernandez, G., Specht, K., Weis, S., Tendolkar, I., Reuber, M., Fell, J., Klaver, P., Ruhlmann, J., Reul, J., and Elger, C. E. (2003). Intrasubject reproducibility of presurgical language lateralization and mapping using fMRI. Neurology 60, 969-975.

FitzGerald, D. B., Cosgrove, G. R., Ronner, S., Jiang, H., Buchbinder, B. R., Belliveau, J. W., Rosen, B. R., and Benson, R. R. (1997). Location of language in the cortex: a comparison between functional MR imaging and electrocortical stimulation. AJNR Am. J. Neuroradiol. 18, 1529-1539.

Floel, A., Jansen, A., Deppe, M., Kanowski, M., Konrad, C., Sommer, J., and Knecht, S. (2005). Atypical hemispheric dominance for attention: functional MRI topography. J. Cereb. Blood Flow Metab. 25, 1197-1208.

Fox, P. T., and Raichle, M. E. (1986). Focal physiological uncoupling of cerebral blood flow and oxidative metabolism during somatosensory stimulation in human subjects. Proc. Natl. Acad. Sci. U.S.A. 83, 1140-1144.

Genovese, C. R., Noll, D. C., and Eddy, W. F. (1997). Estimating test-retest reliability in functional MR imaging. I: statistical methodology. Magn. Reson. Med. 38, 497-507.

Halsey, J. H. (1990). Effect of emitted power on waveform intensity in transcranial Doppler. Stroke 21, 1573-1578.

Hartje, W., Ringelstein, E. B., Kistinger, B., Fabianek, D., and Willmes, K. (1994).
Transcranial Doppler ultrasonic assessment of middle cerebral artery blood flow velocity changes during verbal and visuospatial cognitive tasks. Neuropsychologia 32, 1443-1452.

Henson, R. N., and Friston, K. J. (2007). "Convolution models for fMRI," in Statistical Parametrical Mapping, eds K. J. Friston, J. T. Ashburner, S. J. Kiebel, T. J. Nichols, and W. D. Penny (Amsterdam: Academic Press), 178-192.

Isaacs, K. L., Barr, W. B., Nelson, P. K., and Devinsky, O. (2006). Degree of handedness and cerebral dominance. Neurology 66, 1855-1858.

Jansen, A., Menke, R., Sommer, J., Forster, A. F., Bruchmann, S., Hempleman, J., Weber, B., and Knecht, S. (2006). The assessment of hemispheric lateralization in functional MRI - robustness and reproducibility. Neuroimage 33 , 204-217.

Kim, S. G., and Ugurbil, K. (1997). Comparison of blood oxygenation and cerebral blood flow effects in fMRI: estimation of relative oxygen consumption change. Magn. Reson. Med. 38, 59-65.

Knake, S., Haag, A., Hamer, H. M., Dittmer, C., Bien, S., Oertel, W.H., and Rosenow, F. (2003). Language lateralization in patients with temporal lobe epilepsy: a comparison of functional transcranial Doppler sonography and the Wada test. Neuroimage 19, 1228-1232.

Knecht, S., Deppe, M., Drager, B., Bobe, L., Lohmann, H., Ringelstein, E., and Henningsen, H. (2000a). Language lateralization in healthy right-handers. Brain 123(Pt 1), 74-81.

Knecht, S., Drager, B., Deppe, M., Bobe, L., Lohmann, H., Floel, A., Ringelstein, E. B., and Henningsen, H. (2000b). Handedness and hemispheric language dominance in healthy humans. Brain 123(Pt 12), 2512-2518.

Knecht, S., Deppe, M., Ebner, A., Henningsen, H., Huber, T., Jokeit, H., and Ringelstein, E. B. (1998a). Noninvasive determination of language lateralization by functional transcranial Doppler sonography: a comparison with the Wada test. Stroke 29, 82-86.

Knecht, S., Deppe, M., Ringelstein, E. B., Wirtz, M., Lohmann, H., Drager, B., Huber, T., and Henningsen, $\mathrm{H}$. (1998b). Reproducibility of functional transcranial Doppler sonography in determining hemispheric language lateralization. Stroke 29, 1155-1159.

Knecht, S., Henningsen, H., Deppe, M., Huber, T., Ebner, A., and Ringelstein, E. B. (1996). Successive activation of both cerebral hemispheres during cued word generation. Neuroreport 7 , 820-824.
Kwong, K. K., Belliveau, J. W., Chesler, D. A., Goldberg, I. E., Weisskoff, R. M., Poncelet, B. P., Kennedy, D. N. Hoppel, B. E., Cohen, M. S., and Turner, R. (1992). Dynamic magnetic resonance imaging of human brain activity during primary sensory stimulation. Proc. Natl. Acad. Sci. U.S.A. 89, 5675-5679.

Lewis, R. S., Orsini, D. L., and Satz, P. (1988). Individual differences in the cerebral organization of language using input and output interference measures of lateralization. Arch. Clin. Neuropsychol. 3, 111-119.

Logothetis, N. K., Pauls, J., Augath, M., Trinath, T., and Oeltermann, A. (2001). Neurophysiological investigation of the basis of the fMRI signal. Nature 412, 150-157.

Lohmann, H., Deppe, M., Jansen, A., Schwindt, W., and Knecht, S. (2004). Task repetition can affect functional magnetic resonance imaging-based measures of language lateralization and lead to pseudoincreases in bilaterality. J. Cereb. Blood Flow Metab. 24, 179-187.

McGonigle, D. J., Howseman, A. M., Athwal, B. S., Friston, K. J., Frackowiak, R. S., and Holmes, A. P. (2000). Variability in fMRI: an examination of intersession differences. Neuroimage 11, 708-734.

Njemanze, P. C. (2005). Cerebral lateralization and general intelligence: gender differences in a transcranial Doppler study. Brain Lang. 92, 234-239.

Ogawa, S., Menon, R. S., Tank, D. W. Kim, S. G., Merkle, H., Ellermann, J. M., and Ugurbil, K. (1993). Functional brain mapping by blood oxygenation level-dependent contrast magnetic resonance imaging. A comparison of signal characteristics with a biophysical model. Biophys. J. 64, 803-812.

Oldfield, R. C. (1971). The assessment and analysis of handedness: the Edinburgh inventory. Neuropsychologia 9, 97-113.

Pujol, J., Deus, J., Losilla, J. M., and Capdevila, A. (1999). Cerebral lateralization of language in normal lefthanded people studied by functional MRI. Neurology 52, 1038-1043.

Rasmussen, T., and Milner, B. (1977). The role of early left-brain injury in determining lateralization of cerebral speech functions. Ann. N. Y. Acad. Sci. 299, 355-369.

Rihs, F., Sturzenegger, M., Gutbrod, K., Schroth, G., and Mattle, H. P. (1999). Determination of language dominance: Wada test confirms functional transcranial Doppler sonography. Neurology 52, 1591-1596.

Roux, F. E., Boulanouar, K., Lotterie, J. A., Mejdoubi, M., LeSage, J. P., and
Berry, I. (2003). Language functional magnetic resonance imaging in preoperative assessment of language areas: correlation with direct cortical stimulation. Neurosurgery 52 , 1335-1345.

Rutten, G. J., Ramsey, N. F., van Rijen, P. C., Noordmans, H. J., and van Veelen, C.W. (2002a). Development of a functional magnetic resonance imaging protocol for intraoperative localization of critical temporoparietal language areas. Ann. Neurol. 51, 350-360.

Rutten, G. J., Ramsey, N. F., van Rijen, P. C., and van Veelen, C. W. (2002b). Reproducibility of fMRI-determined language lateralization in individual subjects. Brain Lang. 80, 421-437.

Sommer, I. E., Diederen, K. M., Blom, J.D., Willems, A., Kushan, L., Slotema, K., Boks, M. P., Daalman, K., Hoek, H.W., Neggers, S. F., and Kahn, R. S. (2008). Auditory verbal hallucinations predominantly activate the right inferior frontal area. Brain 131, 3169-3177.

Sommer, I. E. C., and Kahn, R. S. (2009). Language Lateralization and Psychosis. Cambridge: Cambridge University Press.

Springer, J. A., Binder, J. R., Hammeke, T. A., Swanson, S. J., Frost, J. A., Bellgowan, P. S., Brewer, C. C., Perry, H. M., Morris, G. L., and Mueller, W. M. (1999). Language dominance in neurologically normal and epilepsy subjects: a functional MRI study. Brain 122(Pt 11), 2033-2046.

Szaflarski, J. P., Binder, J. R., Possing, E. T., McKiernan, K. A., Ward, B. D., and Hammeke, T.A. (2002). Language lateralization in left-handed and ambidextrous people: fMRI data. Neurology 59, 238-244.

Tomasi, D., Caparelli, E. C., Chang, L., and Ernst, T. (2005). fMRI-acoustic noise alters brain activation during working memory tasks. Neuroimage 27, 377-386.

Tzourio, N., Crivello, F., Mellet, E., Nkanga-Ngila, B., and Mazoyer, B. (1998). Functional anatomy of dominance for speech comprehension in left handers vs right handers. Neuroimage 8, 1-16.

Tzourio-Mazoyer, N., Landeau, B., Papathanassiou, D., Crivello, F., Etard, O., Delcroix, N., Mazoyer, B., and Joliot, M. (2002). Automated anatomical labeling of activations in SPM using a macroscopic anatomical parcellation of the MNI MRI single-subject brain. Neuroimage 15, 273-289.

van der Zwan, A., Hillen, B., Tulleken, C. A., and Dujovny, M. (1993). A quantitative investigation of the variability of the major cerebral arterial territories. Stroke 24, 1951-1959.

van Zijl, P. C., Eleff, S. M., Ulatowski, J. A., Oja, J. M., Ulug, A. M., Traystman, 
R. J., and Kauppinen, R. A. (1998). Quantitative assessment of blood flow, blood volume and blood oxygenation effects in functional magnetic resonance imaging. Nat. Med. 4, 159-167.

Vingerhoets, G., and Stroobant, N. (1999). Lateralization of cerebral blood flow velocity changes during cognitive tasks. A simultaneous bilateral transcranial Doppler study. Stroke 30, 2152-2158.

Wellmer, J., Weber, B., Weis, S., Klaver, P., Urbach, H., Reul, J., Fernandez, G., and Elger, C.E. (2008). Strongly lateralized activation in language fMRI of atypical dominant patients-implications for presurgical work-up. Epilepsy Res. $80,67-76$.

Woermann, F. G., Jokeit, H., Luerding, R., Freitag, H., Schulz, R., Guertler, S., Okujava, M., Wolf, P., Tuxhorn, I., and Ebner, A. (2003). Language lateralization by Wada test and fMRI in 100 patients with epilepsy. Neurology 61, 699-701.

Worsley, K. J., and Friston, K. J. (1995). Analysis of $\mathrm{fMRI}$ time-series revisited - again. Neuroimage 2, 173-181.

Zandbelt, B. B., Gladwin, T. E., Raemaekers, M., van, B. M., Neggers, S. F., Kahn, R. S., Ramsey, N. F., and Vink, M. (2008). Within-subject variation in BOLD-fMRI signal changes across repeated measurements: quantification and implications for sample size. Neuroimage 42, 196-206.

Conflict of Interest Statement: The authors declare that the research was conducted in the absence of any commercial or financial relationships that could be construed as a potential conflict of interest.

Received: 23 November 2010; paper pending published: 05 December 2010; accepted: 10 March 2011; published online: 28 March 2011.
Citation: Somers $M$, Neggers SF, Diederen KM, Boks MP, Kahn RS and Sommer IE (2011) The measurement of language lateralization with functional transcranial Doppler and functional MRI: a critical evaluation. Front. Hum. Neurosci. 5:31. doi: 10.3389/ fnhum.2011.00031

Copyright (c) 2011 Somers, Neggers, Diederen, Boks, Kahn and Sommer. This is an open-access article subject to an exclusive license agreement between the authors and Frontiers Media SA, which permits unrestricted use, distribution, and reproduction in any medium, provided the original authors and source are credited. 